Pacific

Journal of

Mathematics

HARMONIC FORMS ON MANIFOLDS WITH WEIGHTED POINCARÉ INEQUALITY

Jui-Tang Ray Chen and Chiung-Jue Anna Sung

Volume $242 \quad$ No. 2

October 2009 


\title{
HARMONIC FORMS ON MANIFOLDS WITH WEIGHTED POINCARÉ INEQUALITY
}

\author{
Jui-TAng Ray Chen and Chiung-Jue Anna Sung
}

\begin{abstract}
Let $M$ be a complete noncompact manifold. We prove vanishing and finiteness results for harmonic $p$-forms on $M$, assuming both the curvature operator lower bound and the weighted Poincaré inequality on $M$.
\end{abstract}

\section{Introduction}

It is interesting to study the structures of noncompact complete manifolds, especially their topological properties at infinity. There are many results [Witten and Yau 1999; Cai and Galloway 1999; Wang 2001; 2001; Wan and Xin 2004] on the topology of conformally compact manifolds. Recently, by assuming that the Ricci curvature is bounded from below in terms of the dimension and of the first eigenvalue, Li and Wang [2001] obtained information on the topology of complete manifolds infinity and in some cases the metric structure of these manifolds, by proving a vanishing-type theorem of $L^{2}$ harmonic 1-forms. In his thesis, Lam [2007] generalized Li and Wang's result by relaxing the curvature assumptions. He proved that a manifold must have finitely many nonparabolic ends if a similar inequality between the Ricci curvature and the weight function in the weighted Poincaré inequality (see Definition 1.1) is valid outside a compact subset.

In this note, we will consider general harmonic $p$-forms. Working with a complete manifold $M$ satisfying a weighted Poincaré inequality and a curvature operator lower bound expressed in terms of the dimension and the weight function, we prove vanishing and finiteness theorems for the $L^{d}$ harmonic $p$-forms. Also, on an end of manifold with weighted $p$-Poincaré inequality, we prove that the Green's form satisfies a sharp decay estimate. Let us first recall some definitions.

Definition 1.1. Let $M^{m}$ be an $m$-dimensional complete Riemannian manifold. We say that $M^{m}$ satisfies a weighted Poincaré inequality [Li and Wang 2006] with a

MSC2000: primary 58A10; secondary 53C21.

Keywords: weighted Poincaré inequality, harmonic forms.

Both authors were partially supported by NSC Taiwan. 
nonnegative weight function $\rho(x)$ if the inequality

$$
\int_{M} \rho(x) \phi^{2} \leq \int_{M}|\nabla \phi|^{2} \quad \text { for } \phi \in C_{0}^{\infty}(M)
$$

is valid for all compactly supported smooth functions $\phi \in C_{0}^{\infty}(M)$.

Definition 1.2. Let $M^{m}$ be an $m$-dimensional complete Riemannian manifold. We say that $M^{m}$ has property $\left(\mathscr{P}_{\rho}\right)$ if a weighted Poincaré inequality is valid on $M$ with some nonnegative weight function $\rho$ and if the $\rho$-metric, defined by $d s_{\rho}^{2}=\rho d s_{M}^{2}$, is complete.

Let $\lambda_{1}(M)$ denote the greatest lower bound of the spectrum of the Laplacian acting on $L^{2}$ functions. Then the variation principle for $\lambda_{1}(M)$ asserts the validity of the Poincaré inequality

$$
\lambda_{1}(M) \int_{M} \phi^{2} \leq \int_{M}|\nabla \phi|^{2}
$$

for all compactly supported functions $\phi \in C_{0}^{\infty}(M)$. If $\lambda_{1}(M)$ is positive, then obviously $M$ has property $\left(\mathscr{P}_{\rho}\right)$ with $\rho(x)=\lambda_{1}(M)$. Property $\left(\mathscr{P}_{\rho}\right)$ may be seen as a generalization of the assumption $\lambda_{1}(M)>0$.

For harmonic $p$-forms, let $C_{0}^{\infty}\left(\bigwedge^{p} M\right)$ denote the space of smooth $p$-forms with compact support on $M$. Then we define property $\left(\mathscr{P}_{p, \rho}\right)$ as follows.

Definition 1.3. Let $M^{m}$ be an $m$-dimensional complete Riemannian manifold. We say that $M^{m}$ has property $\left(\mathscr{P}_{p, \rho}\right)$ if a weighted $p$-Poincaré inequality is valid on $M$ with some nonnegative weight function $\rho$, that is,

$$
\int_{M} \rho(x)|\phi|^{2} \leq \int_{M}|d \phi|^{2}+|\delta \phi|^{2} \quad \text { for } \phi \in C_{0}^{\infty}\left(\bigwedge^{p} M\right),
$$

and if the $\rho$-metric, defined by $d s_{\rho}^{2}=\rho d s_{M}^{2}$, is complete.

If the greatest lower bound $\lambda_{1, p}$ of the $p$ spectrum satisfies $\lambda_{1, p}(M)>0$, then $M$ has property $\left(\mathscr{P}_{p, \rho}\right)$ with the weight function $\rho(x)=\lambda_{1, p}(M)$. Hence property $\left(\mathscr{P}_{p, \rho}\right)$ can also be viewed as a generalization of the assumption that $\lambda_{1, p}(M)>0$.

Throughout, we use $H_{d}^{p}\left(M^{m}\right)$ to denote the space of $L^{d}$ harmonic $p$-forms, and $r_{\rho}(x)$ to denote the geodesic distance from some fixed point to $x$ with respect to the metric $d s_{\rho}^{2}$. Our main result is the following.

Theorem 1.4. For $m \geq 3$, let $M^{m}$ be a complete noncompact Riemannian manifold with properties $\left(\mathscr{P}_{\rho_{0}}\right)$ and $\left(\mathscr{P}_{p, \rho}\right)$. Suppose the volume growth of $M$ satisfies

$$
\int_{B_{\rho}(2 R) \backslash B_{\rho}(R)} \rho e^{-2(m-p-1) r_{\rho}} \leq c R
$$


and the curvature operator $K_{p}$ of $M^{m}$ has the lower bound

$$
K_{p}>-\frac{m-p}{m-p-1} \rho_{0} \quad \text { on } M .
$$

Then the space $H_{d}^{p}(M)$ is trivial for $2 \leq d \leq 2(m-p-1) /(m-p-2)$.

We will employ some of the arguments from [ $\mathrm{Li}$ and Wang 2001] to prove the sharp decay estimate for the Green's form in the next section, and prove the main theorem in Section 3.

\section{Decay estimate}

Let $M^{m}$ be a complete manifold, and let $\Delta$ be the Hodge Laplace-Beltrami operator of $M^{m}$ acting on differential $p$-forms. The Weitzenböck formula gives

$$
\Delta=\nabla^{2}-K_{p},
$$

where $\nabla^{2}$ is the Bochner Laplacian and $K_{p}$ is an endomorphism depending upon the curvature tensor of $M^{m}$. Using an orthonormal basis $\left\{\theta^{1}, \ldots, \theta^{m}\right\}$ dual to $\left\{e_{1}, \ldots, e_{m}\right\}$, one may express the curvature term $K_{p}$ as

$$
\left\langle K_{p}(w), w\right\rangle=\left\langle\sum_{j, k=1}^{m} \theta^{k} \wedge i_{e_{j}} R\left(e_{k}, e_{j}\right) w, w\right\rangle .
$$

In particular, $\left\langle K_{1}(w), w\right\rangle=\operatorname{Ric}\left(w^{\sharp}, w^{\sharp}\right)$, where $w^{\sharp}$ is the vector dual to the form $w$. We say $M^{m}$ has curvature lower bound $k_{p}$ if for all $p$-forms $w$ on $M^{m}$,

$$
\left\langle K_{p}(w), w\right\rangle \geq k_{p}|w|^{2}
$$

We recall [Li 2000] that an end is simply an unbounded component of $M \backslash D$, where $D$ is a compact smooth domain of $M$. Write $E(R)=E \cap B_{q}(R)$, and define $\partial E(R)=\partial E \cup\left(\partial B_{q}(R) \cap E\right)$. Let $\lambda_{1, p}(E(R))$ be the first eigenvalue of $\Delta$ for $p$-forms satisfying Dirichlet boundary conditions on $\partial E(R)$, that is, $\lambda_{1, p}(E)=$ $\inf _{R>0, E(R) \neq \varnothing} E(R)$; see [Donnelly 1984; Donnelly and Xavier 1984]. Therefore we have for all $w \in C_{0}^{\infty}\left(\bigwedge^{p} E\right)$

$$
\lambda_{1, p}(E) \int|w|^{2} \leq \int\left(|\nabla w|^{2}+\left\langle K_{p}(w), w\right\rangle\right),
$$

where $C_{0}^{\infty}\left(\bigwedge^{p} E\right)$ is the space of smooth $p$-forms with compact supported on the end $E$. If the $p$-spectrum $\lambda_{1, p}(E)$ is positive, then $E$ has property $\left(\mathscr{P}_{p, \rho}\right)$ with the weight function $\rho=\lambda_{1, p}(E)$.

In this section, we study the harmonic $p$-forms on the end $E$ of a manifold with weighted $p$-Poincaré inequality and prove the following decay estimate. See [Donnelly 1984; Li and Wang 2001]. 
Lemma 2.1. Let $M$ be a complete noncompact manifold. If $E$ is an end of $M$ with the property $\left(\mathscr{P}_{p, \rho}\right)$ for some nonnegative weight function $\rho$, then for any smooth harmonic p-form $w$ satisfying

$$
\int_{E_{\rho}(2 R) \backslash E_{\rho}(R)} \rho(x) \exp \left(-2 r_{\rho}\right)|w|^{2} d v=o(R),
$$

we have

$$
\begin{array}{r}
\int_{E_{\rho}(R)} \rho(x) \exp \left(2 r_{\rho}\right)|w|^{2} d v \leq C R, \\
\int_{E_{\rho}(R+1) \backslash E_{\rho}(R)} \rho(x) \exp \left(2 r_{\rho}\right)|w|^{2} d v \leq C
\end{array}
$$

for all $R$ sufficiently large and for some constant $C$ depending on $w$ and $\rho$.

Proof. Let $\psi$ be a nonnegative cutoff function. Integration by parts gives

$$
\begin{aligned}
\int_{E}|\nabla(\psi w)|^{2} & +\left\langle K_{p}(\psi w), \psi w\right\rangle \\
& =\int_{E}|\nabla \psi|^{2}|w|^{2}+2 \psi\langle w \nabla \psi, \nabla w\rangle+|\psi|^{2}|\nabla w|^{2}+\psi^{2}\left\langle K_{p}(w), w\right\rangle \\
& =\int_{E}|\nabla \psi|^{2}|w|^{2}-\int_{E} \psi^{2}\langle w, \Delta w\rangle_{M} \\
& =\int_{E}|\nabla \psi|^{2}|w|^{2} .
\end{aligned}
$$

By property $\left(\mathscr{P}_{p, \rho}\right)$,

$$
\int_{E} \rho(x) \psi^{2}|w|^{2} d v \leq \int_{E}\left(|\nabla(\psi w)|^{2}+\left\langle K_{p}(\psi w), \psi w\right\rangle\right),
$$

so we have

$$
\int_{E} \rho(x) \psi^{2}|w|^{2} d v \leq \int_{E}|\nabla \psi|^{2}|w|^{2} d v
$$

for any cutoff function $\psi$ on $E$. Let $\psi=\phi\left(r_{\rho}(x)\right) \exp \left(a\left(r_{\rho}(x)\right)\right)$. Then

(3) $\int_{E} \phi^{2} \exp (2 a)|w|^{2} d v \leq \int_{E}\left(|\nabla \phi|^{2}+2\langle\nabla \phi, \nabla a\rangle \phi+|\nabla a|^{2} \phi^{2}\right) \exp (2 a)|w|^{2} d v$.

Choose $\phi$ as the nonnegative cutoff function defined by

$$
\phi\left(r_{\rho}(x)\right)= \begin{cases}r_{\rho}(x)-R_{0} & \text { on } E_{\rho}\left(R_{0}+1\right) \backslash E_{\rho}\left(R_{0}\right), \\ 1 & \text { on } E_{\rho}(R) \backslash E_{\rho}\left(R_{0}+1\right), \\ \left(2 R-r_{\rho}(x)\right) / R & \text { on } E_{\rho}(2 R) \backslash E_{\rho}(R), \\ 0 & \text { on } E \backslash E_{\rho}(2 R),\end{cases}
$$


and also choose $a\left(r_{\rho}(x)\right)$ as

$$
a\left(r_{\rho}(x)\right)= \begin{cases}\delta r_{\rho}(x) & \text { for } r_{\rho} \leq K /(1+\delta), \\ K-r_{\rho}(x) & \text { for } r_{\rho}>K /(1+\delta),\end{cases}
$$

for some fixed $K>\left(R_{0}+1\right)(1+\delta)$ and $0<\delta<1$. If $R \geq K /(1+\delta)$, it is easy to check that

$$
\begin{aligned}
& |\nabla \phi|^{2}(x)= \begin{cases}\rho(x) & \text { on } E_{\rho}\left(R_{0}+1\right) \backslash E_{\rho}\left(R_{0}\right), \\
0 & \text { on } E_{\rho}(R) \backslash E_{\rho}\left(R_{0}+1\right), \\
\rho(x) / R^{2} & \text { on } E_{\rho}(2 R) \backslash E_{\rho}(R), \\
0 & \text { on } E \backslash E_{\rho}(2 R),\end{cases} \\
& |\nabla a|^{2}(x)= \begin{cases}\delta^{2} \rho(x) & \text { for } r \leq K /(1+\delta), \\
\rho(x) & \text { for } r>K /(1+\delta),\end{cases}
\end{aligned}
$$

and then

$$
\langle\nabla \phi, \nabla a\rangle(x)= \begin{cases}\delta \rho(x) & \text { on } E_{\rho}\left(R_{0}+1\right) \backslash E_{\rho}\left(R_{0}\right), \\ \rho(x) / R & \text { on } E_{\rho}(2 R) \backslash E_{\rho}(R), \\ 0 & \text { otherwise. }\end{cases}
$$

Therefore (3) becomes

$$
\begin{aligned}
& \int_{E_{\rho}(2 R)} \rho \phi^{2} \exp (2 a)|w|^{2} d v \\
& \leq \int_{E_{\rho}(2 R)}\left(|\nabla \phi|^{2}+2\langle\nabla \phi, \nabla a\rangle \phi+|\nabla a|^{2} \phi^{2}\right) \exp (2 a)|w|^{2} d v \\
& \leq \quad \int_{E_{\rho}\left(R_{0}+1\right) \backslash E_{\rho}\left(R_{0}\right)} \rho \exp (2 a)|w|^{2} d v+\frac{1}{R^{2}} \int_{E_{\rho}(2 R) \backslash E_{\rho}(R)} \rho \exp (2 a)|w|^{2} d v \\
& \quad+2 \delta \int_{E_{\rho}\left(R_{0}+1\right) \backslash E_{\rho}\left(R_{0}\right)} \rho \phi \exp (2 a)|w|^{2} d v+\frac{2}{R} \int_{E_{\rho}(2 R) \backslash E_{\rho}(R)} \rho \phi \exp (2 a)|w|^{2} d v \\
& \quad+\delta^{2} \int_{E_{\rho}\left(\frac{K}{1+\delta}\right) \backslash E_{\rho}\left(R_{0}\right)} \rho \phi^{2} \exp (2 a)|w|^{2} d v+\int_{E_{\rho}(2 R) \backslash E_{\rho}\left(\frac{K}{1+\delta}\right)} \rho \phi^{2} \exp (2 a)|w|^{2} d v .
\end{aligned}
$$

Hence

$$
\begin{aligned}
& \left(1-\delta^{2}\right) \int_{E_{\rho}\left(\frac{K}{1+\delta}\right) \backslash E_{\rho}\left(R_{0}+1\right)} \rho \exp (2 a)|w|^{2} d v \\
& \leq\left(\delta^{2}+2 \delta+1\right) \int_{E_{\rho}\left(R_{0}+1\right) \backslash E_{\rho}\left(R_{0}\right)} \rho \exp (2 a)|w|^{2} d v \\
& \quad+\left(\frac{1}{R^{2}}+\frac{2}{R}\right) \int_{E_{\rho}(2 R) \backslash E_{\rho}(R)} \rho \exp (2 a)|w|^{2} d v .
\end{aligned}
$$


By the definition of $a(x)$ and the growth estimate of $w$ (see (1)), the last term on the right side tends to 0 as $R \rightarrow \infty$. Thus we obtain the estimate

$$
\frac{1-\delta^{2}}{(\delta+1)^{2}} \int_{E_{\rho}\left(\frac{K}{1+\delta}\right) \backslash E_{\rho}\left(R_{0}+1\right)} \rho \exp \left(2 \delta r_{\rho}\right)|w|^{2} d v \leq \int_{E_{\rho}\left(R_{0}+1\right) \backslash E_{\rho}\left(R_{0}\right)} \rho \exp \left(2 \delta r_{\rho}\right)|w|^{2} d v .
$$

Since the right side of this inequality is independent of $K$, by letting $K \rightarrow \infty$ we conclude that

$$
\int_{E \backslash E_{\rho}\left(R_{0}+1\right)} \rho \exp \left(2 \delta r_{\rho}\right)|w|^{2} d v \leq C \quad \text { for some constant } 0<C<\infty .
$$

Next we improve this estimate by setting $a\left(r_{\rho}(x)\right)=r_{\rho}(x)$ in the preceding argument. For $R_{0}<R_{1}<R$, let us choose $\phi$ to be

$$
\phi(x)= \begin{cases}\left(r_{\rho}(x)-R_{0}\right) /\left(R_{1}-R_{0}\right) & \text { on } E_{\rho}\left(R_{1}\right) \backslash E_{\rho}\left(R_{0}\right), \\ \left(R-r_{\rho}(x)\right) /\left(R-R_{1}\right) & \text { on } E_{\rho}(R) \backslash E_{\rho}\left(R_{1}\right) .\end{cases}
$$

The inequality (3) asserts that

$$
\begin{aligned}
& \int_{E_{\rho}(R)} \rho \phi^{2} \exp \left(2 r_{\rho}\right)|w|^{2} d v \leq \int_{E_{\rho}(R)}\left|\nabla\left(\phi \exp \left(r_{\rho}\right)\right)\right|^{2}|w|^{2} d v \\
&=\frac{1}{\left(R-R_{1}\right)^{2}} \int_{E_{\rho}(R) \backslash E_{\rho}\left(R_{1}\right)} \rho \exp \left(2 r_{\rho}\right)|w|^{2} d v \\
&+\frac{1}{\left(R_{1}-R_{0}\right)^{2}} \int_{E_{\rho}\left(R_{1}\right) \backslash E_{\rho}\left(R_{0}\right)} \rho \exp \left(2 r_{\rho}\right)|w|^{2} d v \\
&-\frac{2}{\left(R-R_{1}\right)^{2}} \int_{E_{\rho}(R) \backslash E_{\rho}\left(R_{1}\right)}\left(R-r_{\rho}(x)\right) \rho \exp \left(2 r_{\rho}\right)|w| k 2 d v \\
&+\frac{2}{\left(R_{1}-R_{0}\right)^{2}} \int_{E_{\rho}\left(R_{1}\right) \backslash E_{\rho}\left(R_{0}\right)}\left(r_{\rho}(x)-R_{0}\right) \rho \exp \left(2 r_{\rho}\right)|w|^{2} d v \\
&+\int_{E_{\rho}(R)} \rho \phi^{2} \exp \left(2 r_{\rho}\right)|w|^{2} d v .
\end{aligned}
$$

Then

$$
\begin{aligned}
& \frac{2}{\left(R-R_{1}\right)^{2}} \int_{E_{\rho}(R) \backslash E_{\rho}\left(R_{1}\right)}\left(R-r_{\rho}(x)\right) \rho \exp \left(2 r_{\rho}\right)|w|^{2} d v \\
& \leq \frac{1}{\left(R-R_{1}\right)^{2}} \int_{E_{\rho}(R) \backslash E_{\rho}\left(R_{1}\right)} \rho \exp \left(2 r_{\rho}\right)|w|^{2} d v \\
& \quad+\frac{1}{\left(R_{1}-R_{0}\right)^{2}} \int_{E_{\rho}\left(R_{1}\right) \backslash E_{\rho}\left(R_{0}\right)} \rho \exp \left(2 r_{\rho}\right)|w|^{2} d v \\
& \quad+\frac{2}{\left(R_{1}-R_{0}\right)} \int_{E_{\rho}\left(R_{1}\right) \backslash E_{\rho}\left(R_{0}\right)} \frac{r_{\rho}(x)-R_{0}}{R_{1}-R_{0}} \rho \exp \left(2 r_{\rho}\right)|w|^{2} d v .
\end{aligned}
$$


On the other hand, for any fixed $0<t<R-R_{1}$, $\frac{t}{\left(R-R_{1}\right)^{2}} \int_{E_{\rho}(R-t) \backslash E_{\rho}\left(R_{1}\right)} \rho \exp \left(2 r_{\rho}\right)|w|^{2} d v$

$$
\leq \frac{1}{\left(R-R_{1}\right)^{2}} \int_{E_{\rho}(R) \backslash E_{\rho}\left(R_{1}\right)}\left(R-r_{\rho}(x)\right) \rho \exp \left(2 r_{\rho}\right)|w|^{2} d v,
$$

we deduce that

$$
\begin{aligned}
\frac{2 t}{\left(R-R_{1}\right)^{2}} \int_{E_{\rho}(R-t) \backslash E_{\rho}\left(R_{1}\right)} \rho \exp \left(2 r_{\rho}\right)|w|^{2} d v & \\
\leq\left(\frac{1}{\left(R_{1}-R_{0}\right)^{2}}\right. & \left.+\frac{2}{R_{1}-R_{0}}\right) \int_{E_{\rho}\left(R_{1}\right) \backslash E_{\rho}\left(R_{0}\right)} \rho \exp \left(\sqrt{2} r_{\rho}\right)|w|^{2} d v \\
& +\frac{1}{\left(R-R_{1}\right)^{2}} \int_{E_{\rho}(R) \backslash E_{\rho}\left(R_{1}\right)} \rho \exp \left(\sqrt{2} r_{\rho}\right)|w|^{2} d v .
\end{aligned}
$$

Observe that if $R_{1}=R_{0}+1$, if $t=1$, and if

$$
g(R)=\int_{E_{\rho}(R) \backslash E_{\rho}\left(R_{0}+1\right)} \rho \exp \left(2 r_{\rho}\right)|w|^{2} d v,
$$

then the inequality (5) can be written as

$$
g(R-1) \leq C_{1} R^{2}+\frac{1}{2} g(R),
$$

where $C_{1}=2 \int_{E_{\rho}\left(R_{0}+1\right) \backslash E_{\rho}\left(R_{0}\right)} \rho \exp \left(2 r_{\rho}\right)|w|^{2} d v$ is independent of $R$. Iterating this inequality, we show that for any positive integer $k, R \geq 1$, and constant $C_{2}$,

$$
\begin{aligned}
g(R) & \leq C_{1} \sum_{i=1}^{k} \frac{(R+i)^{2}}{2^{i-1}}+2^{-k} g(R+k) \\
& \leq C_{1} R^{2} \sum_{i=1}^{\infty} \frac{(1+i)^{2}}{2^{i-1}}+2^{-k} g(R+k) \leq C_{2} R^{2}+2^{-k} g(R+k) .
\end{aligned}
$$

However, the previous estimate in (4) asserts that

$$
\int_{E} \rho \exp \left(2 \delta r_{\rho}\right)|w|^{2} d v \leq C \quad \text { for any } \delta<1 .
$$

This implies that

$$
\begin{aligned}
g(R+k) & =\int_{E_{\rho}(R+2 k) \backslash E_{\rho}\left(R_{0}+1\right)} \rho \exp \left(2 r_{\rho}\right)|w|^{2} \\
& \leq \exp (2(1-\delta)(R+k)) \int_{E_{\rho}(R+k) \backslash E_{\rho}\left(R_{0}+1\right)} \rho \exp \left(2 \delta r_{\rho}\right)|w|^{2} \\
& \leq C \exp (2(1-\delta)(R+k)) .
\end{aligned}
$$


Hence $2^{-k} g(R+k) \rightarrow 0$ as $k \rightarrow \infty$ by choosing $2(1-\delta)<\ln 2$. This proves the estimate that $g(R) \leq C_{2} R^{2}$. Adjusting the constant, we have

$$
\int_{E_{\rho}(R)} \rho \exp \left(2 r_{\rho}\right)|w|^{2} d v \leq C_{3} R^{2} \quad \text { for all } R \geq R_{0}
$$

Using inequality (5) again and choosing $R_{1}=R_{0}+1, R>2 R_{1}$ and $t=R / 2$, we conclude that

$$
R \int_{E_{\rho}(R / 2) \backslash E_{\rho}\left(R_{0}+1\right)} \rho \exp \left(2 r_{\rho}\right)|w|^{2} d v \leq C_{4} R^{2}+2 \int_{E_{\rho}(R) \backslash E_{\rho}\left(R_{0}+1\right)} \rho \exp \left(2 r_{\rho}\right)|w|^{2} d v .
$$

However, applying the estimate (6) to the second term on the right side, we have

$$
\int_{E_{\rho}(R / 2) \backslash E_{\rho}\left(R_{0}+1\right)} \rho \exp \left(2 r_{\rho}\right)|w|^{2} d v \leq C_{5} R .
$$

Therefore

$$
\int_{E_{\rho}(R)} \rho \exp \left(2 r_{\rho}\right)|w|^{2} d v \leq C_{6} R \quad \text { for } R \geq R_{0}
$$

We are ready to prove the theorem by using (7). Setting $t=2$ and $R_{1}=R-4$ in (5), we have

$$
\begin{aligned}
& \int_{E_{\rho}(R-2) \backslash E_{\rho}(R-4)} \rho \exp \left(2 r_{\rho}\right)|w|^{2} d v \\
& \leq\left(\frac{4}{\left(R-R_{0}-4\right)^{2}}+\frac{8}{R-R_{0}-4}\right) \int_{E_{\rho}(R-4) \backslash E_{\rho}\left(R_{0}\right)} \rho \exp \left(2 r_{\rho}\right)|w|^{2} d v \\
&+\frac{1}{4} \int_{E_{\rho}(R) \backslash E_{\rho}(R-4)} \rho \exp \left(2 r_{\rho}\right)|w|^{2} d v .
\end{aligned}
$$

According to (7), the first term of the right side is bounded by a constant. Hence this inequality can be rewritten as

$$
\int_{E_{\rho}(R-2) \backslash E_{\rho}(R-4)} \rho \exp \left(2 r_{\rho}\right)|w|^{2} d v \leq C_{7}+\frac{1}{2} \int_{E_{\rho}(R) \backslash E_{\rho}(R-4)} \rho \exp \left(2 r_{\rho}\right)|w|^{2} d v .
$$

Iterating this inequality $k$ times, we have

$$
\begin{aligned}
& \int_{E_{\rho}(R+2) \backslash E_{\rho}(R)} \rho \exp \left(2 r_{\rho}\right)|w|^{2} d v \\
& \leq C_{7} \sum_{i=0}^{k-1} 2^{-i}+\frac{1}{2^{k}} \int_{E_{\rho}(R+2(k+1)) \backslash E_{\rho}(R)} \rho \exp \left(2 r_{\rho}\right)|w|^{2} d v .
\end{aligned}
$$


Using (7) again, we conclude the second term is bounded by

$$
\frac{1}{2^{k}} \int_{E_{\rho}(R+2(k+1)) \backslash E_{\rho}(R)} \rho \exp \left(2 r_{\rho}\right)|w|^{2} d v \leq \frac{C(R+2(k+1))}{2^{k}},
$$

and the upper bound tends to zero as $k \rightarrow \infty$. Hence

$$
\int_{E_{\rho}(R+2) \backslash E_{\rho}(R)} \rho \exp \left(2 r_{\rho}\right)|w|^{2} d v \leq C_{8}
$$

for some constant $C_{8}>0$ independent of $R$.

Corollary 2.2. Let $M$ be a complete manifold. If $E$ is an end of $M$ with positive $\lambda(E)$, where $\lambda(E)$ is equal to either $\lambda_{1, p}(E)$ or $\lambda_{1}(E)+K_{p}$, then for any smooth harmonic p-form $w$ satisfying

$$
\int_{E(2 R) \backslash E(R)} \exp (-2 \sqrt{\lambda(E)} r)|w|^{2} d v=o(R),
$$

we have

$$
\begin{array}{r}
\int_{E(R)} \exp (2 \sqrt{\lambda(E)} r)|w|^{2} d v \leq C R, \\
\int_{E(R+1) \backslash E(R)} \exp (2 \sqrt{\lambda(E)} r)|w|^{2} d v \leq C,
\end{array}
$$

for all $R$ sufficiently large.

\section{Vanishing and finiteness theorems of harmonic $p$-forms}

Let $w$ be a harmonic $p$-form on an $m$-dimensional manifold $M$. Then $w$ satisfies the Kato inequality [Wan and Xin 2004; Calderbank et al. 2000; Herzlich 2000]

$$
|\nabla w|^{2} \geq \frac{m-p+1}{m-p}|\nabla| w||^{2},
$$

and equality holds if and only if there exists a 1-form $\alpha$ with $\alpha \wedge w=0$ such that

$$
\nabla w=\alpha \otimes w-\frac{1}{m+1-p} \sum_{j=1}^{m} \theta^{j} \otimes\left(\theta^{j} \wedge i_{\alpha^{\sharp}} w\right),
$$

where $\left\{\theta^{1}, \ldots, \theta^{m}\right\}$ is an orthonormal basis for the cotangent bundle and $\alpha^{\sharp}$ is the vector dual to $\alpha$.

Now we are ready to prove vanishing and finiteness theorems for harmonic $p$ forms using the decay estimate Lemma 2.1 and the Kato inequality. To simplify our statement, we will assume the function $\rho$ is bounded in the rest of the section. 
Theorem 3.1. For $m \geq 3$, let $M^{m}$ be a complete noncompact Riemannian manifold with properties $\left(\mathscr{P}_{\rho_{0}}\right)$ and $\left(\mathscr{P}_{p, \rho}\right)$. Suppose the volume growth of $M$ satisfies

$$
\int_{B_{\rho}(2 R) \backslash B_{\rho}(R)} \rho e^{-2(m-p-1) r_{\rho}} \leq c R
$$

and the curvature operator $K_{p}$ of $M^{m}$ has the lower bound

$$
K_{p} \geq-\frac{m-p}{m-p-1} \rho_{0} \quad \text { on } M .
$$

Then any harmonic $p$-form $w$ in $L^{d}$ with $2 \leq d \leq 2(m-p-1) /(m-p-2)$ must either vanish or satisfy Equation (8).

Proof. The theorem is obviously true if $d=2$ as $\left(\mathscr{P}_{p, \rho}\right)$ holds on $M$. So we assume $d>2$. Let $w$ be a smooth harmonic $p$-form. By the Kato inequality, the Bochner formula becomes $|w| \Delta|w| \geq \frac{1}{m-p}|\nabla| w||^{2}+K_{p}|w|^{2}$.

Let $g=|w|^{(m-p-1) /(m-p)}$. Then this inequality can be rewritten as

$$
\Delta g \geq \frac{m-p-1}{m-p} K_{p} g .
$$

We first show that $g$ satisfies the integral estimate $\int_{B_{\rho}(2 R) \backslash B_{\rho}(R)} \rho g^{2} \leq C R$. To see this, using the Schwarz inequality, we have

$$
\begin{gathered}
\int_{B_{\rho}(2 R) \backslash B_{\rho}(R)} \rho g^{2} \leq\left(\int_{B_{\rho}(2 R) \backslash B_{\rho}(R)} \rho \exp \left(2 r_{\rho}\right)|w|^{2}\right)^{m-p-1 /(m-p)} \\
\cdot\left(\int_{B_{\rho}(2 R) \backslash B_{\rho}(R)} \rho \exp \left(-2(m-p-1) r_{\rho}\right)\right)^{1 /(m-p)} .
\end{gathered}
$$

By the volume growth condition, the second term on the right side satisfies

$$
\int_{B_{\rho}(2 R) \backslash B_{\rho}(R)} \rho \exp \left(-2(m-p-1) r_{\rho}\right) \leq c R .
$$

On the other hand, for $a=d /(d-2)$, we have

$$
\begin{aligned}
\int_{B_{\rho}(2 R) \backslash B_{\rho}(R)} \rho \exp \left(-2 r_{\rho}\right)|w|^{2} & \leq\left(\int_{B_{\rho}(2 R) \backslash B_{\rho}(R)} \rho^{a} \exp \left(-2 a r_{\rho}\right)\right)^{1 / a} \\
& \leq C\left(\int_{B_{\rho}(2 R) \backslash B_{\rho}(R)} \rho \exp \left(-2(m-p-1) r_{\rho}\right)\right)^{1 / a} \\
& \leq C R^{1 / a},
\end{aligned}
$$


since $w$ is in $L^{d}$. Now according to Lemma 2.1, one has

$$
\int_{B_{\rho}(2 R) \backslash B_{\rho}(R)} \rho \exp \left(2 r_{\rho}\right)|w|^{2} \leq C R .
$$

Then (10) can be written as $\int_{B_{\rho}(2 R) \backslash B_{\rho}(R)} \rho g^{2} \leq C R$.

To finish the proof of the theorem, note that for a cutoff function $\phi$, we have

$$
\int_{M} \rho_{0} \phi^{2} g^{2} \leq \int_{M}|\nabla(\phi g)|^{2}=\int_{M}|\nabla \phi|^{2} g^{2}+2 \phi g\langle\nabla \phi, \nabla g\rangle+|\phi|^{2}|\nabla g|^{2} .
$$

Also,

$$
\int_{M} 2 \phi g\langle\nabla \phi, \nabla g\rangle=\int_{M} g\left\langle\nabla \phi^{2}, \nabla g\right\rangle=-\int_{M} \phi^{2}|\nabla g|^{2}-\int_{M} \phi^{2} g \Delta g .
$$

Therefore

$$
\int_{M} \rho_{0} \phi^{2} g^{2} \leq \int_{M}|\nabla \phi|^{2} g^{2}-\int_{M} \phi^{2} g \Delta g
$$

or in other words,

$$
\int_{M} \phi^{2} g\left(\rho_{0} g+\Delta g\right) \leq \int_{M}|\nabla \phi|^{2} g^{2} .
$$

Let us now choose $\phi=\phi\left(r_{\rho}\right)$ to satisfy the properties that

$$
\phi= \begin{cases}1 & \text { on } B_{\rho}(R), \\ 0 & \text { on } M \backslash B_{\rho}(2 R),\end{cases}
$$

and

$$
\left|\phi^{\prime}(t)\right| \leq 2 R^{-1} \text { for } R \leq t \leq 2 R .
$$

Then

$$
\int_{B_{\rho}(2 R)} \phi^{2} g^{2}\left(\rho_{0}+\frac{m-p-1}{m-p} K_{p}\right) \leq C R^{-2} \int_{B_{\rho}(2 R) \backslash B_{\rho}(R)} \rho g^{2} .
$$

The right side of this tends to zero as $R \rightarrow \infty$. Since $K_{p} \geq-(m-p) \rho_{0} /(m-p-1)$, we conclude that $g$ must be identically zero, or

$$
\Delta g=\frac{m-p-1}{m-p} K_{p} g .
$$

This in particular implies that $w$ must satisfy (8).

Next we prove the finiteness theorem for the space of harmonic $p$-forms if the curvature lower bound only holds on $M \backslash B_{q}\left(R_{0}\right)$, where $B_{q}\left(R_{0}\right)$ is a geodesic ball in $M$. 
Theorem 3.2. For $m \geq 3$, let $M^{m}$ be a complete noncompact Riemannian manifold with properties $\left(\mathscr{P}_{\rho_{0}}\right)$ and $\left(\mathscr{P}_{p, \rho}\right)$. Suppose the volume growth of $M$ satisfies

$$
\int_{B_{\rho}(2 R) \backslash B_{\rho}(R)} \rho e^{-2(m-p-1) r_{\rho}} \leq c R
$$

and the curvature operator $K_{p}$ of $M^{m}$ has the lower bound

$$
K_{p} \geq-\left(\frac{m-p}{m-p-1}-\epsilon\right) \rho_{0} \quad \text { on } M \backslash B_{q}\left(R_{0}\right) .
$$

Then $\operatorname{dim} H_{d}^{p}(M) \leq C\left(m, p, \epsilon, B_{q}\left(R_{0}\right)\right)$ with $2 \leq d \leq 2(m-p-1) /(m-p-2)$.

Proof. According to the proof of the vanishing Theorem 3.1, for each $w \in H_{d}^{p}$, function $g=|w|^{2(m-p-1) /(m-p)}$ satisfies the estimate

$$
\int_{B_{\rho}(2 R) \backslash B_{\rho}(R)} g^{2} \leq C R .
$$

Also, the Bochner formula together with the curvature assumption implies that the function $g$ satisfies the differential inequality

$$
\Delta g \geq\left(\frac{m-p-1}{m-p} \epsilon-\rho_{0}\right) g \quad \text { on } M \backslash B_{q}\left(R_{0}\right) .
$$

Let $\phi$ be a cutoff function satisfying

$$
\begin{aligned}
\phi & = \begin{cases}0 & \text { on } B_{q}\left(R_{0}\right), \\
1 & \text { on } B_{\rho}(R) \backslash B_{q}\left(2 R_{0}\right), \\
0 & \text { on } M \backslash B_{\rho}(2 R),\end{cases} \\
|\nabla \phi| & \leq C R_{0}^{-1} \quad \text { on } B_{q}\left(2 R_{0}\right) \backslash B_{q}\left(R_{0}\right), \\
|\nabla \phi| \leq C \sqrt{\rho} R^{-1} & \text { on } B_{\rho}(2 R) \backslash B_{\rho}(R)
\end{aligned}
$$

for some constant $C>0$.

Since

$$
\int_{M} \rho_{0} \phi^{2} g^{2} \leq \int_{M}|\nabla(\phi g)|^{2}=\int_{M}|\nabla \phi|^{2} g^{2}+2 \phi g\langle\nabla \phi, \nabla g\rangle+|\phi|^{2}|\nabla g|^{2}
$$

and

$$
\int_{M} 2 \phi g\langle\nabla \phi, \nabla g\rangle=\int_{M} g\left\langle\nabla \phi^{2}, \nabla g\right\rangle=-\int_{M} \phi^{2}|\nabla g|^{2}-\int_{M} \phi^{2} g \Delta g,
$$

we conclude

$$
\int_{M} \rho_{0} \phi^{2} g^{2} \leq \int_{M}|\nabla \phi|^{2} g^{2}-\int_{M} \phi^{2} g \Delta g
$$


Hence, we have

$$
\int_{M \backslash B_{q}\left(R_{0}\right)} \phi^{2} g\left(\rho_{0} g+\Delta g\right) \leq C R_{0}^{-2} \int_{B_{q}\left(2 R_{0}\right) \backslash B_{q}\left(R_{0}\right)} g^{2}+C R^{-2} \int_{B_{\rho}(2 R) \backslash B_{\rho}(R)} \rho g^{2} .
$$

Let $R \rightarrow \infty$. Then

$$
\frac{m-p-1}{m-p} \epsilon \int_{M \backslash B_{q}\left(2 R_{0}\right)} g^{2} \leq C R_{0}^{-2} \int_{B_{q}\left(2 R_{0}\right) \backslash B_{q}\left(R_{0}\right)} g^{2} .
$$

In particular,

$$
\int_{B_{q}\left(3 R_{0}\right)} g^{2} \leq\left(1+\frac{C}{\epsilon R_{0}^{2}}\right) \int_{B_{q}\left(2 R_{0}\right)} g^{2}
$$

It is now standard to conclude [Li 1980] that $\operatorname{dim} H_{d}^{p} \leq C$.

\section{References}

[Cai and Galloway 1999] M. Cai and G. J. Galloway, "Boundaries of zero scalar curvature in the AdS/CFT correspondence”, Adv. Theor. Math. Phys. 3 (1999), 1769-1783 (2000). MR 2002k:53080 Zbl 0978.53084

[Calderbank et al. 2000] D. M. J. Calderbank, P. Gauduchon, and M. Herzlich, "Refined Kato inequalities and conformal weights in Riemannian geometry", J. Funct. Anal. 173:1 (2000), 214-255. MR 2001f:58046 Zbl 0960.58010

[Donnelly 1984] H. Donnelly, "Eigenforms of the Laplacian on complete Riemannian manifolds", Comm. Partial Differential Equations 9:13 (1984), 1299-1321. MR 86c:58145 Zbl 0552.58027

[Donnelly and Xavier 1984] H. Donnelly and F. Xavier, "On the differential form spectrum of negatively curved Riemannian manifolds", Amer. J. Math. 106:1 (1984), 169-185. MR 85i:58115 Zbl 0547.58034

[Herzlich 2000] M. Herzlich, "Refined Kato inequalities in Riemannian geometry", in Journées “Équations aux Dérivées Partielles” (La Chapelle sur Erdre, 2000), Exposé 6, Univ. Nantes, 2000. MR 2001i:58068 Zbl 1028.58025

[Lam 2007] L. Lam, Weighted Poincaré inequality and manifolds with spin holonomy, thesis, University of California at Irvine, 2007.

[Li 1980] P. Li, "On the Sobolev constant and the $p$-spectrum of a compact Riemannian manifold", Ann. Sci. École Norm. Sup. (4) 13:4 (1980), 451-468. MR 82h:58054 Zbl 0466.53023

[Li 2000] P. Li, "Curvature and function theory on Riemannian manifolds", pp. 375-432 in Surveys in differential geometry, edited by S.-T. Yau, Surv. Differ. Geom. 7, Int. Press, Somerville, MA, 2000. MR 2003g:53047 Zbl 1066.53084

[Li and Wang 2001] P. Li and J. Wang, "Complete manifolds with positive spectrum", J. Differential Geom. 58:3 (2001), 501-534. MR 2003e:58046 Zbl 1032.58016

[Li and Wang 2006] P. Li and J. Wang, "Weighted Poincaré inequality and rigidity of complete manifolds", Ann. Sci. École Norm. Sup. 39:6 (2006), 921-982. MR 2008d:53053 Zbl 1120.53018

[Wan and Xin 2004] T. Y. H. Wan and Y. L. Xin, "Vanishing theorems for conformally compact manifolds", Comm. Partial Differential Equations 29:7-8 (2004), 1267-1279. MR 2005h:53058 Zbl 1086.53060 
[Wang 2001] X. Wang, "On conformally compact Einstein manifolds", Math. Res. Lett. 8:5-6 (2001), 671-688. MR 2003d:53075 Zbl 1053.53030

[Witten and Yau 1999] E. Witten and S.-T. Yau, "Connectedness of the boundary in the AdS/CFT correspondence”, Adv. Theor. Math. Phys. 3:6 (1999), 1635-1655 (2000). MR 2002b:53071 Zbl 0978.53085

Received October 20, 2008. Revised March 21, 2009.

JUI-TANG RAY CHEN

DEPARTMENT OF MATHEMATICS

NATIONAL TAIWAN NORMAL UNIVERSITY

88 SECTION 4

TING CHOU ROAD

TAIPEI

TAIWAN

jtchen@math.ntnu.edu.tw

Chiung-Jue AnNa Sung

National Tsing Hua University

DEPARTMENT OF MATHEMATICS

Number 101, SECTION 2

GUANGFU ROAD

HSINCHU 30013

TAIWAN

cjsung@math.nthu.edu.tw 